\title{
Multilayer graphenes with mixed stacking structure: Interplay of Bernal and rhombohedral stacking
}

\author{
Mikito Koshino \\ Department of Physics, Tohoku University, Sendai, 980-8578, Japan \\ Edward McCann \\ Department of Physics, Lancaster University, Lancaster, LA1 4YB, United Kingdom
}

(Received 21 November 2012; published 22 January 2013)

\begin{abstract}
We study the electronic structure of multilayer graphenes with a mixture of Bernal and rhombohedral stacking and propose a general scheme to understand the electronic band structure of an arbitrary configuration. The system can be viewed as a series of finite Bernal graphite sections connected by stacking faults. We find that the low-energy eigenstates are mostly localized in each Bernal section, and, thus, the whole spectrum is well approximated by a collection of the spectra of independent sections. The energy spectrum is categorized into linear, quadratic, and cubic bands corresponding to specific eigenstates of Bernal sections. The ensemble-averaged spectrum exhibits a number of characteristic discrete structures originating from finite Bernal sections or their combinations likely to appear in a random configuration. In the low-energy region, in particular, the spectrum is dominated by frequently appearing linear bands and quadratic bands with special band velocities or curvatures. In the higher-energy region, band edges frequently appear at some particular energies, giving optical absorption edges at the corresponding characteristic photon frequencies.
\end{abstract}

DOI: 10.1103/PhysRevB.87.045420

PACS number(s): 73.22.Pr, 81.05.ue, 73.43.Cd

\section{INTRODUCTION}

Graphene multilayers exhibit a wide variety of stacking arrangements allowed by weak van der Waals interlayer coupling, offering various types of quasiparticles in the low-energy electronic spectrum. In three-dimensional bulk graphite, there are two distinct crystal configurations called Bernal $(A B A B \cdots),{ }^{1-6}$ and rhombohedral $(A B C A B C \cdots)$ stacking $^{7-9}$ as illustrated in Fig. 1. A sequence such as $A B C \cdots$ represents the lattice point on every layer along a perpendicular axis, where $A$ and $B$ are inequivalent sublattices of hexagonal lattice, and $C$ is the center of the hexagon. Recently, several experimental techniques such as optical absorption, ${ }^{10-13}$ Raman spectroscopy, ${ }^{14,15}$ and transmission electron microscopy ${ }^{16}$ have been applied to identify the number of layers and the stacking order of graphene multilayers. Few-layer graphene samples exfoliated from bulk graphite usually exhibit Bernal structure, which is supposed to be the most stable, but often also display rhombohedral structure in part. $^{15,16}$

The electronic band structure of graphene multilayer depends sensitively on its stacking structure. ${ }^{15-23}$ In Bernal stacked multilayer, the spectrum consists of quadratic bands analogous to bilayer graphene and a single linear band-like monolayer. ${ }^{24-29}$ In contrast, a rhombohedral-stacked multilayer has a totally different spectrum with a pair of flat low-energy bands, which disperse as $p^{N}$ with momentum $p$ and the number of layers $N .^{24,30-33}$

In general, graphitic structures are expected to take a Bernal-rhombohedral mixed form as illustrated in Fig. 2. The energy spectrum of mixed multilayer was studied for some specific few-layer cases, ${ }^{25,28,32}$ but general rules predicting the electronic properties of arbitrary structure are not well known. A single rhombohedral stacking fault appearing in Bernal graphite was studied theoretically, ${ }^{34}$ and it supports cubic bands associated with the localized states bound to the stacking fault. It was also shown ${ }^{32}$ that the low-energy spectrum of Bernal-rhombohedral mixed multilayer consists of energy bands, which disperse as $p^{J}$, and the sum of $J$ coincides with the number of layers. This predicts the number of the bands belonging to each $J$, but to obtain the quantitative dispersions and wave functions one would need to actually calculate eigenenergies for every single configuration.

In this paper, we study the electronic structures of general Bernal-rhombohedral mixed graphene multilayers. We begin by proposing a general scheme in which to understand the band property of any given configuration without resorting to diagonalizing the full Hamiltonian. We view the system as a series of finite-layered Bernal sections connected by the rhombohedral-type stacking fault, as depicted in Fig. 3(a), and treat the coupling between neighboring sections as a perturbation. We find that the eigenstates near the Dirac point are mostly localized in each Bernal section, and the states are approximated well by those of incomplete Bernal graphites as illustrated in Figs. 3(b) and 3(c). The energy spectrum is then categorized into linear, quadratic and cubic (or higher-order) bands within the basis of incomplete Bernal graphite sections. We also specify several limited situations where the states of neighboring Bernal sections are strongly hybridized.

To model realistic experimental systems with many layers and, perhaps, a number of stacking faults, we develop a statistical approach. To study the electronic properties averaged over different stacking configurations, we analyze the statistics of the velocity of the linear bands and the effective mass of the quadratic bands that dominate the low-energy spectrum. We find that there are some particular frequently-appearing values in the velocity/mass distribution, corresponding to finitelayered Bernal sections and their particular combinations. We also compute the averaged optical absorption spectrum and 
(a) Bernal (ABAB...)

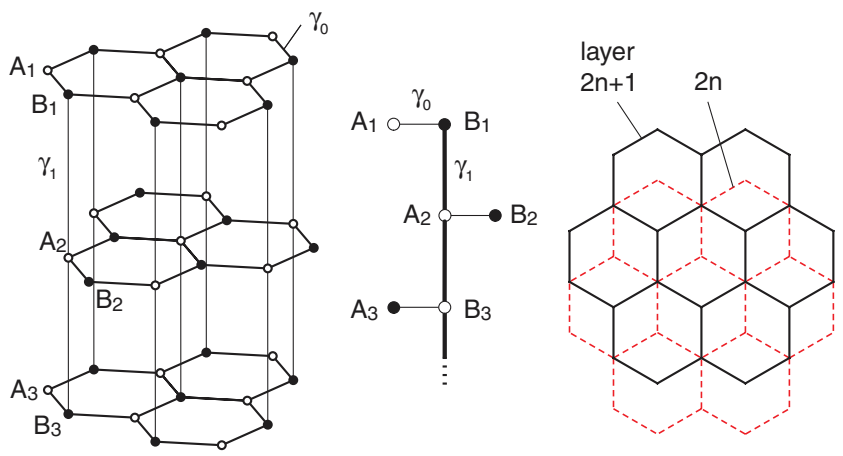

(b) Rhombohedral (ABCABC...)

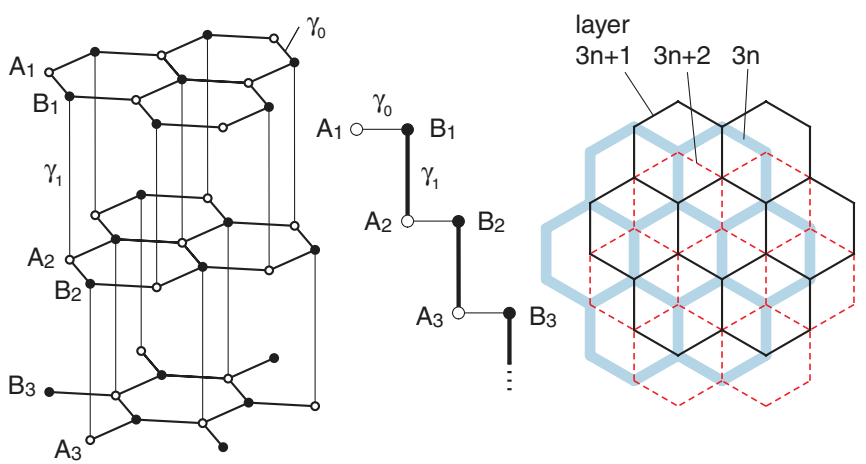

FIG. 1. (Color online) Lattice structures of (a) Bernal and (b) rhombohedral graphites. In each panel, the right figure is a top view, the middle is a schematic diagram of the lattice structure.

find that the absorption edges emerge at particular frequencies, corresponding to frequently-appearing band structures.

The paper is organized as follows. We formulate the Hamiltonian of mixed multilayer graphene in Sec. II. We describe the eigenstates and the energy spectrum of isolated incomplete Bernal graphite section, and study the intersection mixing effect in Sec. III. We present the ensemble-averaged distribution of low-energy band velocity and effective mass, and the optical absorption in Sec. IV.

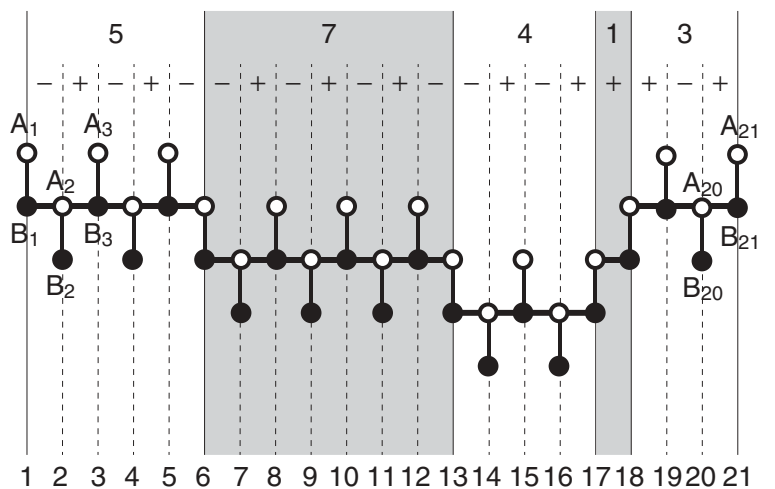

FIG. 2. Example of Bernal-rhombohedral mixed graphene multilayers expressed by $(5,7,4,1,3)$. Intralayer coupling by parameter $\gamma_{0}$ is shown as a vertical solid line, interlayer coupling by $\gamma_{1}$ is shown as a horizontal solid line. (a)

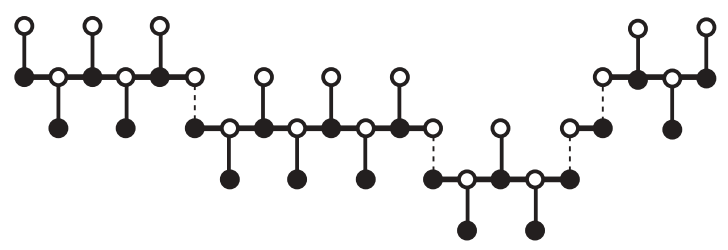

(b)

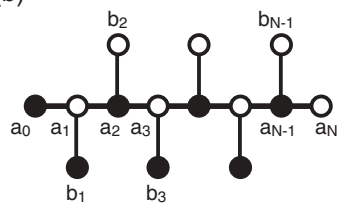

(c)

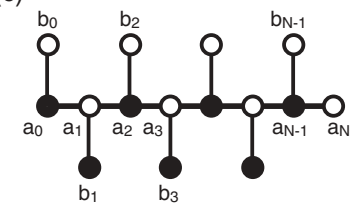

FIG. 3. (a) Decomposition of Bernal-rhombohedral mixed multilayers into incomplete Bernal sections. Dashed lines are connections between neighboring sections, which are to be switched off in the decomposition. (b) Decomposed incomplete Bernal section in the middle and (c) at one end of the whole system.

\section{FORMULATION AND EFFECTIVE-MASS HAMILTONIAN}

We consider an $N_{\text {tot }}$-layer graphene system, which has a unit cell containing $A_{j}$ and $B_{j}$ sublattices on the $j$ th layer. Coupling between the $j$ th and $(j+1)$ th layers is described as either $A B$ or $B A$ stacking, where $A B$ stacking is defined as the arrangement in which sites $A_{j}$ and $B_{j+1}$ are connected by the vertical interlayer coupling, whereas $B A$ stacking as the one in which $B_{j}$ and $A_{j+1}$ are connected. A typical situation is illustrated in Fig. 2, following the schematics for pure Bernal and rhombohedral graphites. ${ }^{24}$ The entire system is specified by a set of indices $\sigma_{j}= \pm$ for $j=1,2, \ldots, N_{\text {tot }}-1$ describing the interlayer connection between $j$ th and $(j+1)$ th layers, where + and - represent $A B$ and $B A$ stacking, respectively. Bernal-stacked graphene is then expressed as an alternating sequence like $(+,-,+,-, \cdots)$, while rhombohedral-stacked graphene is $(+,+,+, \cdots)$ or $(-,-,-, \cdots)$.

In a general configuration, an alternative sequence $(\cdots,+,-,+,-, \cdots)$ is regarded as a section of continuous Bernal structure, and a position at which the same sign $(\cdots,+,+, \cdots)$ or $(\cdots,-,-, \cdots)$ occurs consecutively is regarded as a rhombohedral-type stacking fault separating different Bernal sections. The sequence $\left\{\sigma_{i}\right\}$ is alternatively expressed as a set of integers

$$
\left(N_{1}, N_{2}, N_{3}, \ldots, N_{M}\right),
$$

where $N_{i}$ is the length of the $i$ th Bernal section, and the stacking fault exists between $N_{i}$ and $N_{i+1}$. For example, the sequence $(+,-,+,-,-,+,-,-,+)$ is written as $(4,3,2) . M$ is the number of separated sections in the whole system. The total number of layers in the system is given by

$$
N_{\text {tot }}=1+\sum_{i=1}^{M} N_{i} .
$$

A pure Bernal-stacked multilayer graphene is represented by a single number $\left(N_{\text {tot }}-1\right)$, and a pure rhombohedral multilayer is by $(1,1,1, \ldots)$. 
To describe the electronic properties, we use an effective-

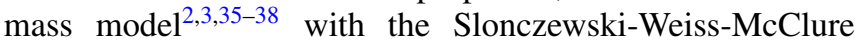
parametrization of graphite. ${ }^{6}$ As the simplest approximation, we include parameter $\gamma_{0}$ describing the nearest neighbor coupling within each layer, and $\gamma_{1}$ for the coupling of the interlayer vertical bonds. The band parameters were experimentally estimated in the bulk $A B A$ graphite, for example, ${ }^{6}$ as $\gamma_{0}=3.16 \mathrm{eV}$ and $\gamma_{1}=0.39 \mathrm{eV}$. The low-energy spectrum is given by states in the vicinity of the $K_{\xi}$ point at the corner of the Brillouin zone, where $\xi= \pm 1$ is the valley index. If $\left|A_{j}\right\rangle$ and $\left|B_{j}\right\rangle$ are Bloch functions at the $K_{\xi}$ point, corresponding to the $A$ and $B$ sublattices of layer $j$, respectively, then, in the basis of $\left|A_{1}\right\rangle,\left|B_{1}\right\rangle,\left|A_{2}\right\rangle,\left|B_{2}\right\rangle, \cdots$, the Hamiltonian in the vicinity of the $K_{\xi}$ valley is

$$
H=\left(\begin{array}{cccccc}
H_{0} & V_{1} & & & & \\
V_{1}^{\dagger} & H_{0} & V_{2} & & & \\
& V_{2}^{\dagger} & H_{0} & V_{3} & & \\
& & V_{3}^{\dagger} & H_{0} & V_{4} & \\
& & & \ddots & \ddots & \ddots
\end{array}\right),
$$

with

$$
\begin{gathered}
H_{0}=\left(\begin{array}{cc}
0 & v \pi^{\dagger} \\
v \pi & 0
\end{array}\right), \\
V_{j}=\left\{\begin{array}{cc}
\left(\begin{array}{cc}
0 & 0 \\
\gamma_{1} & 0
\end{array}\right) & \left(\sigma_{j}=-\right), \\
\left(\begin{array}{cc}
0 & \gamma_{1} \\
0 & 0
\end{array}\right) & \left(\sigma_{j}=+\right) .
\end{array}\right.
\end{gathered}
$$

Here, the in-plane momentum operator is $\pi=\xi \hat{p_{x}}+i \hat{p_{y}}$, and $\hat{\mathbf{p}}=\left(\hat{p}_{x}, \hat{p}_{y}\right)=-i \hbar \nabla$. The diagonal blocks, Eq. (4), describe nearest-neighbor intralayer hopping, and $V$, Eq. (5), describes nearest-neighbor interlayer hopping. $v$ is the band velocity of monolayer graphene given by $v=\sqrt{3} a \gamma_{0} / 2 \hbar$, where $a \approx 0.246 \mathrm{~nm}$ is the lattice constant of the honeycomb lattice. We neglect other hopping parameters in the following arguments for simplicity. Additional effects caused by the neglected parameters will be briefly described in Sec. V.

\section{ELECTRONIC STRUCTURE AND BAND CLASSIFICATION}

Unlike a pure Bernal multilayer, the Hamiltonian of a general graphene stack cannot be simply decomposed into smaller subsystems by a unitary transformation. At small momentum, however, we can show that every eigenstate is almost well localized in a single Bernal-graphite section between rhombohedral stacking faults, so that the system can be treated approximately as a set of independent Bernal sections.

In order to develop an analytical description, we begin by considering an imaginary system in which the intralayer hopping is switched off only on the stacking-fault layers, as illustrated in Fig. 3(a). The entire system is then broken into a set of incomplete Bernal graphite sections with two inequivalent sublattice sites per layer but with one sublattice missing at the stacking fault layers, as illustrated in Figs. 3(b) and 3(c) for a middle section and an end section, respectively. In the following, we will show that at small momentum $p \ll \gamma_{1} / v$, the spectrum is approximately that of a collection of the energy bands of isolated incomplete Bernal graphites, except for some specific occasions where the coupling between different sections is significant.

\section{A. Spectrum of incomplete graphite}

We first consider the electronic structure of an isolated incomplete Bernal graphite of the middle section type, as in Fig. 3(b). For convenience, we divide all the atomic sites into two groups, "chained" and "free" sites, where a chained site refers to a site connected to neighboring layers by $\gamma_{1}$, and a free site is one which has only intralayer connection $\gamma_{0}$. This incomplete Bernal middle section has $N+1$ layers that may be numbered from $j=0$ to $N$. In this respect, it is identical to Bernal-stacked graphite, but, as compared to Bernal-stacked graphite, there is a missing site at each end, and the missing sites are always free sites. We rename the sites $A_{j}$ and $B_{j}$ with $a_{j}$ and $b_{j}$, so that $a_{j}$ and $b_{j}$ represents the chained site and the free site on the $j$-th layer, respectively. If $a_{0}=B_{0}$, for example, the relation is

$$
\begin{aligned}
& a_{j}=\left\{\begin{array}{ll}
B_{j} & (j=\text { even }) \\
A_{j} & (j=\text { odd })
\end{array},\right. \\
& b_{j}=\left\{\begin{array}{cc}
A_{j} & (j=\text { even }) \\
B_{j} & (j=\text { odd })
\end{array} .\right.
\end{aligned}
$$

The Hamiltonian is obtained by eliminating the missing sites $\left(b_{0}\right.$ and $\left.b_{N}\right)$ in complete $N+1$-layer Bernal graphite. As the system includes $2 N$ atoms, there are $2 N$ eigenfunctions at a momentum $\mathbf{p}$, each of which may be expressed as

$$
\left(\begin{array}{l}
\Psi\left(a_{j}\right) \\
\Psi\left(b_{j}\right)
\end{array}\right)=\left(\begin{array}{l}
f\left(a_{j}\right) \\
f\left(b_{j}\right)
\end{array}\right) e^{i \mathbf{p} \cdot \mathbf{x} / \hbar},
$$

where $\mathbf{x}=(x, y)$ is the in-plane position and $\mathbf{p}=\left(p_{x}, p_{y}\right)$ is the in-plane momentum measured from $K_{\xi}$. In this simple model with $\gamma_{0}$ and $\gamma_{1}$, the energy bands are always isotropic around $p=0$, and the dispersion is a function of $p \equiv \sqrt{p_{x}^{2}+p_{y}^{2}}$.

As we describe in the following, it is possible to classify the bases of the eigenfunctions into five categories:

$$
\begin{aligned}
& \text { C1: chained, linear, } \\
& \text { C2: chained, quadratic, } \\
& \text { F1: free, linear, } \\
& \text { F2: free, quadratic, } \\
& \text { F3: free, boundary-localized. }
\end{aligned}
$$

$\mathrm{C}$ and $\mathrm{F}$ represent chained and free sites, respectively. 1 and 2 correspond to the linear and quadratic dispersion in the band structure. F3 gives a dispersion-less flat band in the isolated incomplete graphite, but it forms to a cubic (or higher-order) band when the inter-section coupling is included. As an example, we present in Fig. 4 the band structure and the wave functions of incomplete graphite $N=8$.

The bases of $\mathrm{C} 1$ and $\mathrm{C} 2$ are plane waves on the chained sites, which vanish at imaginary sites $a_{-1}$ and $a_{N+1}$ outside 


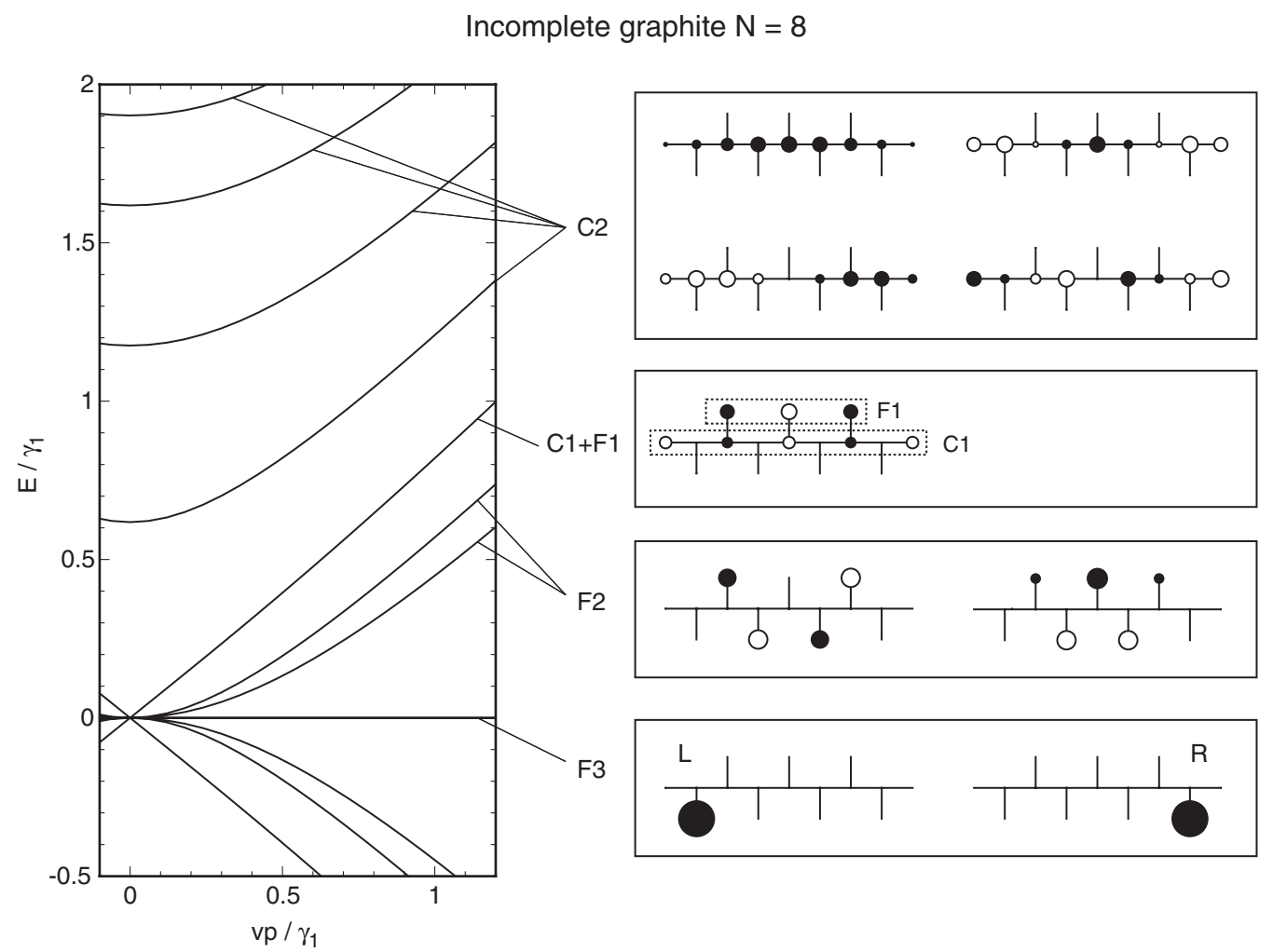

FIG. 4. (Left) Energy bands of incomplete Bernal graphite with $N=8$. (Right) Some of wave functions from different categories at a small momentum along $p_{x}$ direction with $\left|p_{x}\right| \ll \gamma_{1} / v$. White and black circles represent positive and negative wave amplitudes, respectively, and the size is the absolute magnitude.

the system. Explicitly, this is defined as

$$
\left(\begin{array}{l}
f_{q_{l}}^{\mathrm{C}}\left(a_{j}\right) \\
f_{q_{l}}^{\mathrm{C}}\left(b_{j}\right)
\end{array}\right)=\left(\begin{array}{c}
\sqrt{\frac{2}{N+2}} \sin q_{l}(j+1) \\
0
\end{array}\right),
$$

with quantized wave numbers

$$
q_{l}=\frac{l \pi}{N+2}, \quad l=1,2, \ldots, N+1 .
$$

The wave number $q_{l}=\pi / 2$, appearing only when $N$ is even, is categorized in $\mathrm{C} 1$, and all the others in $\mathrm{C} 2$.

The bases of $\mathrm{F} 1$ and $\mathrm{F} 2$ are plane waves on the free sites, but they have nodes at $b_{1}$ and $b_{N-1}$ inside the system. They are written as

$$
\left(\begin{array}{c}
f_{q_{l}^{\prime}}^{\mathrm{F}}\left(a_{j}\right) \\
f_{q_{l}^{\prime}}^{\mathrm{F}}\left(b_{j}\right)
\end{array}\right)=\left(\begin{array}{c}
0 \\
e^{i \theta_{j}} \sqrt{\frac{2}{N-2}} \sin q_{l}^{\prime}(j-1)
\end{array}\right),
$$

with a different series of wave numbers

$$
q_{l}^{\prime}=\frac{l \pi}{N-2}, \quad l=1,2, \ldots, N-3,
$$

where $\theta_{j}$ is defined by $e^{i \theta_{j}}=\left(p_{x} \pm i p_{y}\right) / p$ when $b_{j}=B_{j}$ and $A_{j}$, respectively. F1 or F2 states do not exist when $N \leqslant 3$. The wave number $q_{l}^{\prime}=\pi / 2$, which appears when $N$ is even (more than 4), is categorized in F1, and all the others in F2.
The group F3 is comprised of two states localized at $b_{1}$ or $b_{N-1}$,

$$
\begin{aligned}
\left(\begin{array}{c}
f_{\mathrm{L}}^{\mathrm{F} 3}\left(a_{j}\right) \\
f_{\mathrm{L}}^{\mathrm{F} 3}\left(b_{j}\right)
\end{array}\right) & =\left(\begin{array}{c}
0 \\
\delta_{j, 1}
\end{array}\right), \\
\left(\begin{array}{c}
f_{\mathrm{R}}^{\mathrm{F} 3}\left(a_{j}\right) \\
f_{\mathrm{R}}^{\mathrm{F} 3}\left(b_{j}\right)
\end{array}\right) & =\left(\begin{array}{c}
0 \\
\delta_{j, N-1}
\end{array}\right) .
\end{aligned}
$$

In the case $N=2$, there is a single F3 state localized at the only free site. When $N=1$, there are no F3 states.

At $p=0, \mathrm{C} 1$ states and all $\mathrm{F}$ states (F1, F2, and F3) are the exact eigenstates at zero-energy. Considering the perturbation in $p$ for these degenerate states, the Hamiltonian is approximately block diagonalized into blocks describing $\mathrm{C} 1+\mathrm{F} 1, \mathrm{~F} 2$, and F3 states. $\mathrm{C} 1$ and $\mathrm{F} 1$ are hybridized by a term linear in $p$, to form a monolayer-like Dirac cone,

$$
\varepsilon_{ \pm}^{\mathrm{C} 1+\mathrm{F} 1}(p) \approx \pm \sqrt{\frac{N-2}{N+2}} v p,
$$

but with a reduced band velocity compared to monolayer graphene. ${ }^{32}$ Mixing with other states gives rise to a correction to Eq. (14), but it is quadratic in $p$. The $\mathrm{C} 1+\mathrm{F} 1$ band only appears when $N$ is an even number, because otherwise $\mathrm{C} 1$ or F1 state does not exist. The case of $N=2$ is special, in that $\mathrm{F} 1$ does not exist and $\mathrm{C} 1$ alone gives a flat band at zero energy.

F2 states give low-energy quadratic bands,

$$
\varepsilon_{q_{l}^{\prime}}^{\mathrm{F} 2}(p) \approx-\frac{v^{2} p^{2}}{2 \gamma_{1} \cos q_{l}^{\prime}},
$$


while F3 remains at zero energy independently of $p$,

$$
\varepsilon_{\mathrm{L}}^{\mathrm{F} 3}(p)=\varepsilon_{\mathrm{R}}^{\mathrm{F} 3}(p)=0 .
$$

In Appendix A, we show that F2 and F3 states are actually the approximate eigenstates with the eigenenergies Eqs. (15) and (16), respectively. F2 bands of $q_{l}^{\prime}$ and $\pi-q_{l}^{\prime}$ form a pair of electron and hole bands with the same band mass $m^{*}=$ $\gamma_{1}\left|\cos q_{l}^{\prime}\right| / v^{2}$, analogous to the low-energy bands of bilayer graphene with the mass $m^{*}=\gamma_{1} /\left(2 v^{2}\right){ }^{39}$ Indeed, the $2 \times 2$ effective Hamiltonian spanned by these two F2 states is shown to be equivalent with the low-energy Hamiltonian for bilayer graphene by an appropriate unitary transformation.

C2 states are the eigenstates on the chained sites at $p=$ 0 , with the nonzero eigenenergy $2 \gamma_{1} \cos q_{l}$. For $p \neq 0$, those states are hybridized with the free sites giving rise to quadratic dispersion for $v p \ll 2 \gamma_{1} \cos q_{l}$,

$$
\varepsilon_{q_{l}}^{\mathrm{C} 2}(p) \approx 2 \gamma_{1} \cos q_{l}+\frac{v^{2} p^{2}}{2 \gamma_{1} \cos q_{l}}\left(1-\frac{4}{N+2} \sin ^{2} q_{l}\right) .
$$

This band is electron-type and holetype for $0<q_{l}<\pi / 2$ and $\pi / 2<q_{l}<\pi$, respectively.

In a section located at the end of the whole system as in Fig. 3(c), one of the missing sites, $b_{0}$ or $b_{N}$ is restored, giving a different energy spectrum. When $b_{0}\left(b_{N}\right)$ exists, the plane waves of free sites in Eq. (11) have a node at $b_{-1}\left(b_{N+1}\right)$ (out of the system) instead of $b_{1}\left(b_{N-1}\right)$, so that the quantized wave numbers on F states, Eq. (12), are changed to

$$
q_{l}^{\prime}=\frac{l \pi}{N}, \quad l=1,2, \ldots, N-1 .
$$

The dispersion of the F2 band in Eq. (15) changes according to the new set of $q_{l}^{\prime}$. There are no changes for the $\mathrm{C}$ state wave number in Eq. (10). The linear band of $\mathrm{C} 1+\mathrm{F} 1$, Eq. (14), is modified to

$$
\varepsilon_{ \pm}^{\mathrm{C} 1+\mathrm{F} 1}(p) \approx \pm \sqrt{\frac{N}{N+2}} v p .
$$

For F3 states, either $(\mathrm{F} 3, \mathrm{~L})$ or $(\mathrm{F} 3, \mathrm{R})$, whichever is closer to the end of the whole system, does not exist any more.

When both of $b_{0}$ and $b_{N}$ are restored, the partial system becomes a complete $(N+1)$-layer Bernal graphite, and $q_{l}$ and $q_{l}^{\prime}$ become identical to Eq. (10). Then C2 and F2 bands belonging to $q_{l}$ and $\pi-q_{l}$ compose a subsystem equivalent to bilayer graphene, and the $\mathrm{C} 1+\mathrm{F} 1$ band forms a linear band with band velocity equal to the monolayer's. ${ }^{24,27}$

\section{B. Coupling beyond the stacking faults}

The wave functions of neighboring incomplete graphite sections are generally hybridized at $p \neq 0$ via the bond between the $a_{0}$ site of one section and $a_{N}$ of the other, as illustrated in Fig. 5(a). Particularly strong coupling occurs between the $\mathrm{C} 1+\mathrm{F} 1$ and the $\mathrm{C} 2$ bands because they have significant wave amplitudes at the end sites $a_{0}$ and $a_{N}$,

$$
\left|f_{q_{l}}^{\mathrm{C}}\left(a_{0}\right)\right|=\left|f_{q_{l}}^{\mathrm{C}}\left(a_{N}\right)\right|=\sqrt{\frac{2}{N+2}} \sin q_{l} .
$$

The coupling is generally large at $q_{l}=\pi / 2$ (C1 state), and decreases as $q_{l}$ approaches 0 or $\pi$. The F2 and F3 bands, on
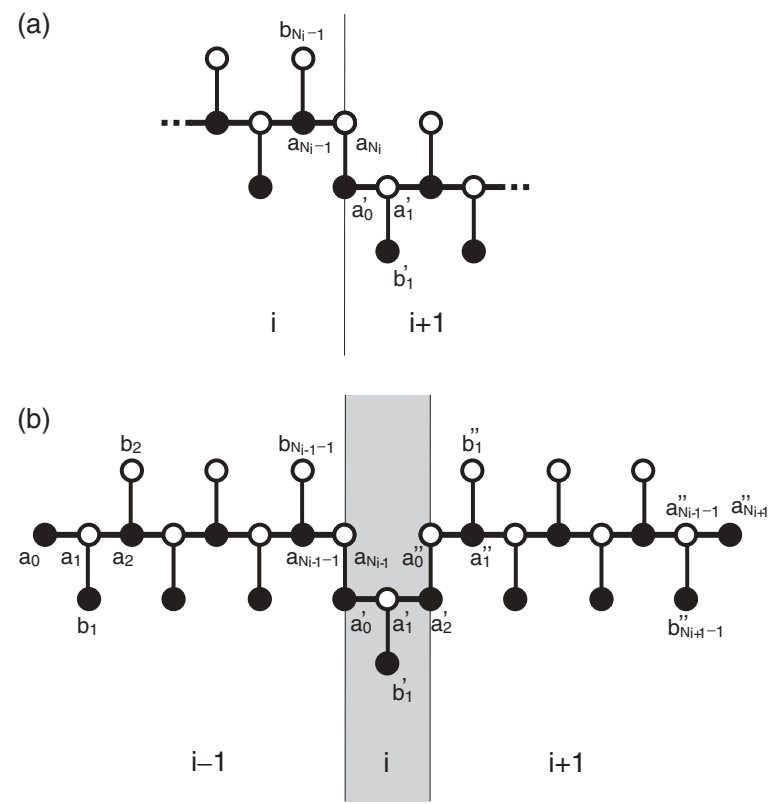

FIG. 5. (a) Schematic of a stacking fault between the neighboring Bernal sections. (b) Structure of $\left(N_{i-1}, 2, N_{i+1}\right)$.

the other hand, are mainly localized on free sites so that the mixing effect is generally small. In the following, we consider details of the intersection hybridization for each eigenstate class.

\section{1. $C 1+F 1$ band}

If the sequence of integers $\left(N_{1}, N_{2}, N_{3}, \ldots, N_{M}\right)$ describing the lengths $N_{i}$ of each Bernal section contains any consecutive even numbers, then the $\mathrm{C} 1+\mathrm{F} 1$ linear bands in those sections are mixed together through the coupling among $\mathrm{C} 1$ states. Let us assume $N_{s}, N_{s+1}, \ldots, N_{s+p}$ are a series of consecutive even numbers. We arrange the bases as $|s, \mathrm{C} 1\rangle,|s, \mathrm{~F} 1\rangle, \ldots, \mid s+$ $p, \mathrm{C} 1\rangle,|s+p, \mathrm{~F} 1\rangle$, where $|i, \mathrm{C} 1\rangle$ represents the $\mathrm{C} 1$ state of the section of $N_{i}$. The Hamiltonian matrix is written for this basis as

$$
H^{\mathrm{C} 1+\mathrm{F} 1}=\left(\begin{array}{ccccc}
h_{s} & u_{s} & & & \\
u_{s}^{\dagger} & h_{s+1} & u_{s+1} & & \\
& u_{s+1}^{\dagger} & h_{s+2} & u_{s+2} & \\
& & \ddots & \ddots & \ddots \\
& & & u_{s+p-1}^{\dagger} & h_{s+p}
\end{array}\right) \text {, }
$$

with

$$
\begin{gathered}
h_{i}=\sqrt{\frac{N_{i}-2\left(1-\delta_{i}\right)}{N_{i}+2}}\left(\begin{array}{cc}
0 & v \pi_{i}^{\dagger} \\
v \pi_{i} & 0
\end{array}\right), \\
u_{i}=\sqrt{\frac{1}{\left(N_{i}+2\right)\left(N_{i+1}+2\right)}}\left(\begin{array}{cc}
v \pi_{i}^{\dagger} & 0 \\
0 & 0
\end{array}\right),
\end{gathered}
$$

where $\pi_{i}=\pi\left(\pi^{\dagger}\right)$ when $a_{0}$ of the section $s$ is $A(B)$ site, and $\delta_{i}=1$ when the section $N_{i}$ is located at the end of the entire system (i.e., $i=1$ or $M$ ), and $\delta_{i}=0$ otherwise. 
Graphene stack $(6,4,1,2)$
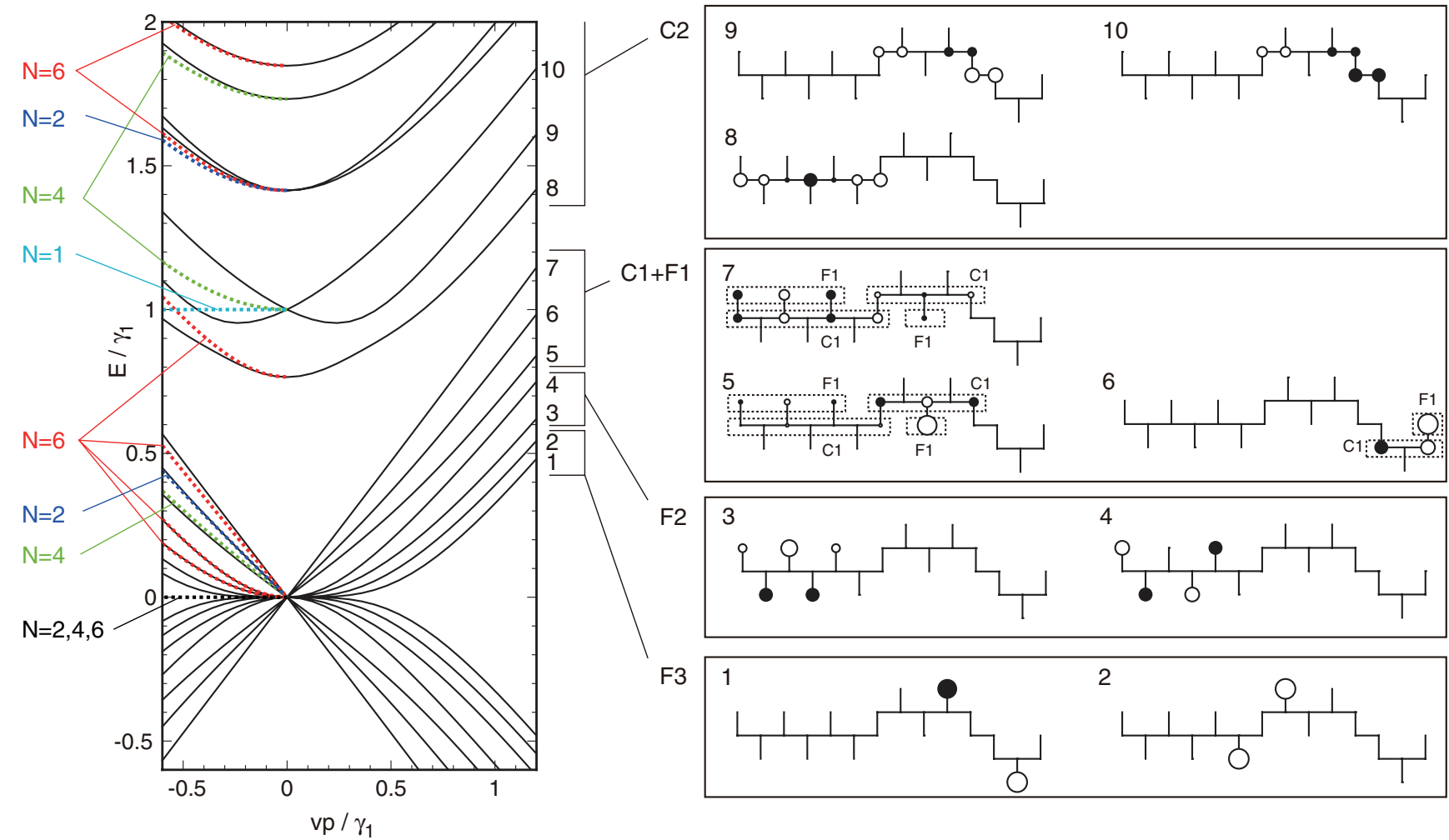

F3

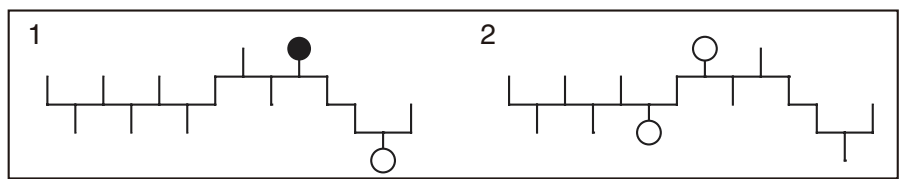

FIG. 6. (Color online) Band structure and some wave functions of the graphene stack $(6,4,1,2)$. Dashed curves plotted for $p<0$ and $E>0$ represent the energy bands of the decomposed incomplete graphites, $N=6,4,1$, and $2(N=6$ and 2 are the end-section type).

The Hamiltonian (21) immediately leads to $p+1$ Dirac cones with generally different band velocities from the original. We found that the velocities are always equal to, or smaller than the monolayer's band velocity $v$, and that velocity equal to $v$ only appears when $N_{s}, \ldots, N_{s+p}$ covers the whole system, i.e., $s=1$ and $s+p=M$.

Figure 6 shows an example of the band structure and the wave functions, calculated for the graphene stack $(6,4,1,2)$. Dashed curves plotted for $p<0$ and $E>0$ represent the energy bands of the decomposed incomplete graphites, $N=$ $6,4,1$, and 2 . We see that the linear bands of the neighboring sections $N=6$ and 4 are hybridized to give the fifth and seventh bands, which have different velocities from the original, while the linear band from $N=2$ is kept almost intact (sixth band).

\section{F2 band}

The coupling between F2 bands in the neighboring sections is proportional to $p^{3}$, because the amplitude at $a_{0}$ and $a_{N}$ is of the order of $p$, and the hopping between $a_{0}$ and $a_{N}$ is $v p$. For small momenta $v p \ll \gamma_{1}$, the coupling effect is negligible compared to the original $\mathrm{F} 2$ band energies $\propto p^{2}$, so that the wave function of F2 states are well localized in each single section.

An exceptionally strong intersection coupling occurs where a section of $N_{i}=2$ exists in the middle of the system, as illustrated in Fig. 5(b). There, C1 states of $N_{i}=2$ strongly hybridize with F2 states of the adjacent sections $i-1$ and $i+1$, and also two F3 states at $b_{N_{i-1}-1}$ and at $b_{1}^{\prime \prime}$ facing to the
$N_{i}=2$. The hybridized eigenenergies are given by Eq. (15) with reconstructed wave numbers $q_{l}^{\prime}$, which are the solutions of

$$
\begin{aligned}
& \sin \left[q_{l}^{\prime}\left(N_{i-1}-1\right)\right] \sin \left[q_{l}^{\prime}\left(N_{i+1}-1\right)\right] \cos ^{2} q_{l}^{\prime} \\
& \quad=\cos \left[q_{l}^{\prime}\left(N_{i-1}-1\right)\right] \cos \left[q_{l}^{\prime}\left(N_{i+1}-1\right)\right] \sin ^{2} q_{l}^{\prime} .
\end{aligned}
$$

If the section $i \pm 1$ is the end section, then $N_{i \pm 1}$ should be replaced with $N_{i \pm 1}+2$. The detailed derivation of Eq. (24) is presented in Appendix B.

\section{F3 band}

Similarly to F2, the coupling between F3 bands in neighboring sections is proportional to $p^{3}$. Since F3 is originally a zero-energy band in an isolated Bernal graphite, the coupling of $O\left(p^{3}\right)$ directly becomes the band dispersion when the intersection coupling is introduced. Let us consider F3 states $|i, \mathrm{~F} 3, \mathrm{R}\rangle$ and $|i+1, \mathrm{~F} 3, \mathrm{~L}\rangle$ in the neighboring sections $i$ and $i+1$, which are facing each other across the stacking fault. We index the sites with $a_{j}, b_{j}$ and $a_{j}^{\prime}, b_{j}^{\prime}$ for the section $i$ and $i+1$, respectively, as illustrated in Fig. 5(a). The state $|i, \mathrm{~F} 3, \mathrm{R}\rangle$ is localized at $b_{N_{i}-1}$, and $|i+1, \mathrm{~F} 3, \mathrm{~L}\rangle$ is at $b_{1}^{\prime}$. When $b_{N_{i}-1}$ is an $A$ site, the effective Hamiltonian in the basis of $|i, \mathrm{~F} 3, \mathrm{R}\rangle$ and $|i+1, \mathrm{~F} 3, \mathrm{~L}\rangle$ becomes

$$
\delta \mathcal{H}^{\text {(eff) }}=\left(\begin{array}{cc}
0 & \left(v \pi^{\dagger}\right)^{3} / \gamma_{1}^{2} \\
(v \pi)^{3} / \gamma_{1}^{2} & 0
\end{array}\right),
$$

leading to cubic dispersion of $\varepsilon= \pm v^{3} p^{3} / \gamma_{1}^{2}$. This actually agrees with the cubic band of the stacking-fault bound states 
argued in Ref. 34. When $b_{N_{i}-1}$ is a $B$-site, $\pi$ and $\pi^{\dagger}$ are interchanged in Eq. (25).

The Hamiltonian (25) is quite similar to that of lowenergy states in rhombohedral trilayer graphene. ${ }^{24,31-33}$ If the stacking faults appear $s$ times consecutively, i.e., $\left(\ldots, N_{i}, 1, \ldots, 1, N_{i+s}, \ldots\right)$, then the states $|i, \mathrm{~F} 3, \mathrm{R}\rangle$ and $\mid i+$ $s, \mathrm{~F} 3, \mathrm{~L}\rangle$ form a Hamiltonian equivalent to a rhombohedral $(s+2)$ layer, giving a $p^{s+2}$ dispersion. In the multilayer $(6,4,1,2)$, we have a pair of $p^{3}$ bands (second band) at the boundary between $N=6$ and 4 , and $p^{4}$ bands (first band) between $N=4$ and 2, as shown in Fig. 6 .

\section{C2 band}

The intersection coupling modifies the band mass of $\mathrm{C} 2$ bands in Eq. (17). The strength of coupling is given by the product of $v p$ and the wave amplitudes at the end sites, Eq. (20). The mass change is irrelevant when $N$ is large $(\gg 1)$ or $\left|q_{l}\right|$ is close to 0 or $\pi$. When the energies of C2 bands in neighboring sections happen to coincide at $p=0$, the two bands are strongly hybridized to give a linear $p$ term in addition to the original quadratic term. This situation always occurs when the same $q_{l}$ is shared by the neighboring sections. In the example of Fig. 6, we see that both of the sections $N=4$ and 1 have a $\mathrm{C} 2$ state of $q_{l}=\pi / 3$, and those two bands are strongly hybridized to the ninth and tenth bands, which are linear at $p=0$.

\section{STATISTICAL PROPERTIES}

\section{A. Linear band velocity and quadratic band mass}

The velocity of linear bands and the effective mass of quadratic bands are important parameters characterizing the low-energy spectrum which can be probed experimentally, for example, by magnetooptical spectroscopy. ${ }^{40,41}$ In Bernalrhombohedral mixed graphene multilayer, there are several frequently appearing values of velocity and mass, each of which is associated with a single incomplete graphite section or particular combinations of them.

For an isolated incomplete graphite, the velocity of linear band $(\mathrm{C} 1+\mathrm{F} 1)$ is given by $\sqrt{(N-2) /(N+2)} v$ for a middle section [see Fig. 3(b)], and $\sqrt{N /(N+2)} v$ for an end section [see Fig. 3(c)]. The effective mass of the quadratic bands (F2) is $\left|2\left(\gamma_{1} / v^{2}\right) \cos q_{l}^{\prime}\right|$, where $q_{l}^{\prime}$ is given by Eqs. (12) and (18) for the middle and end sections, respectively. Figure 7(a) lists the linear band velocity and the quadratic band mass in incomplete graphite sections with some different $N$ 's. Solid and dashed bars represent the values of the middle and end sections, respectively.

Figure 7(b) presents the same quantities of the mixed multilayer stacks of all possible configurations from single layer to seven layers. There, we derived the velocity and the mass from the numerically calculated eigenenergies of the original Hamiltonian (3) at small momenta. In multilayer $(2,3)$, for example, we see that the linear band velocity is actually carried over from an isolated graphite of $N=2$, and the quadratic band mass is from $N=3$ (both of the end-section type), which are listed in Fig. 7(a). For the case where even numbers appear consecutively like $(2,4)$, on the other hand, the linear-band velocities do not coincide with those of the isolated systems as argued in the previous section. The velocities of

TABLE I. (a) List of frequently-appearing velocity of the lowenergy linear band. Symbol $\circ$ in $(\circ, a, b, \circ)$ represents an arbitrary sequence of numbers in which no even number comes next to $a$ or $b$. (b) List of frequently-appearing effective mass of the low-energy quadratic band. Symbol $\bullet$ in $(\bullet, a, b, \bullet)$ represents any sequences in which a number other than 2 comes next to $a$ or $b$.

(a) velocity

(in units of $v$ )

$1 / 2$

$1 \sqrt{3}$

$1 / \sqrt{2}$

$\sqrt{3 / 5}$

$\sqrt{2 / 3}$

$\sqrt{3} / 2$

(b) mass

(in units of $\gamma_{1} / 2 v^{2}$ )

$( \pm 1+\sqrt{5}) / 2$

1

$\sqrt{2}$

$\sqrt{3}$

hybridized linear bands are then calculated by diagonalizing Eq. (21). Similarly, we see that the quadratic band mass does not match with isolated ones, when " 2 " appears somewhere in the middle, as $(1,2,3)$. Then the hybridized mass is given by solving Eq. (24).

The hybridized bands very often have the same band dispersions as a single incomplete graphite section. Tables I(a) and I(b) list typical values of the linear band velocity and the quadratic band mass, respectively, with the corresponding configurations. In the velocity table, the sequence $(\circ, a, b, \circ)$ represents the combination $a, b$ appearing in the middle of the multilayer, and $(a, b, \circ)$ is one located at the end. The symbol o represents an arbitrary sequence of numbers in which no even number comes next to $a$ or $b$ (otherwise the linear bands are hybridized). In the mass table, similarly, • represents any sequences in which a number other than 2 comes next to $a$ and $b$.

\section{B. Statistics of velocity and mass}

A realistic graphene multilayer system is not always uniform in the in-plane direction, but is often composed of many small domains with different stacking structures. ${ }^{16}$ Nevertheless, we expect to observe strong signals from special band velocities or masses as argued above, because they are statistically likely to appear in a random configuration. Here, we consider the distribution of band velocity and mass ensemble-averaged over possible Bernal-rhombohedral mixed configurations. Apart from tight-binding parameters $\gamma_{0}$ and $\gamma_{1}$, the only parameter to characterize the system is $p_{s}$, the probability to have an $A B C$ stacking fault in every layer. Namely, the system is a pure Bernal multilayer when $p_{s}=0$, and pure rhombohedral multilayer when $p_{s}=1$. Experimentally, the value of $p_{s}$ is not well known, and it should depend on the multilayer growing process. From the fact that 
(a) Incomplete graphite

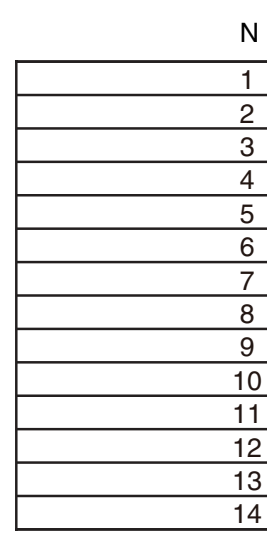

velocity of linear band (in units of $v$ ) mass of quadratic band

(in units of $\gamma_{1} / 2 v^{2}$ )
0.5
10
0.5
1.5
2

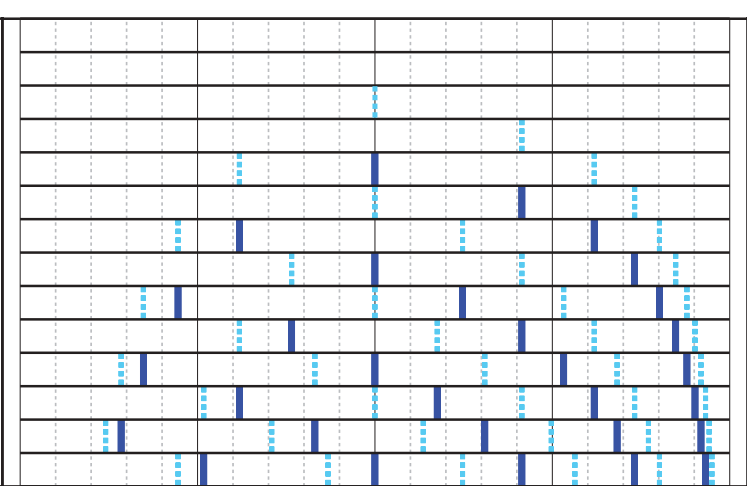

(b) General graphene stack

velocity of linear band (in units of $v$ ) mass of quadratic band (in units of $\gamma_{1} / 2 v^{2}$ )

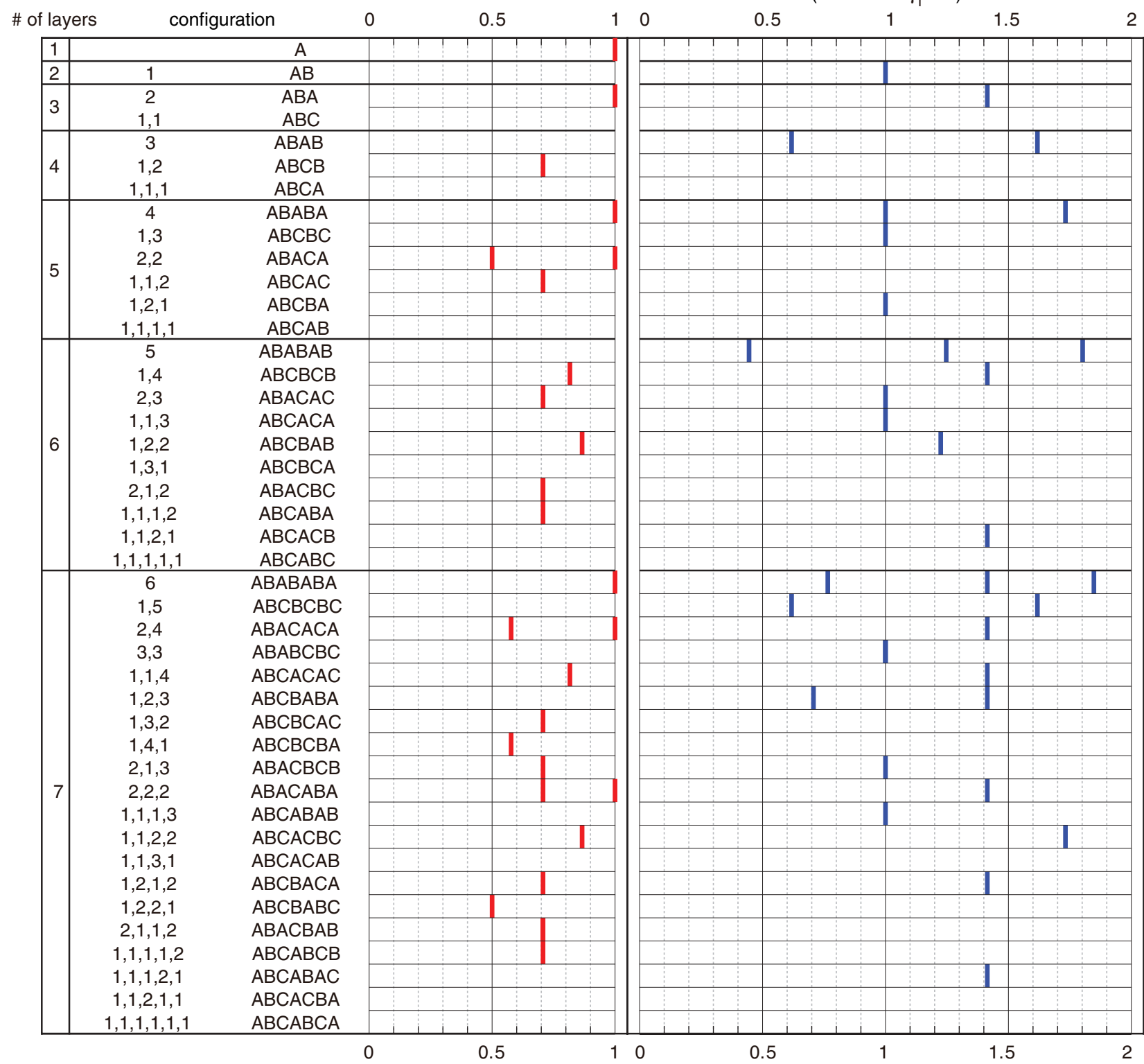

FIG. 7. (Color online) (a) Lists of the linear band velocity and the quadratic band mass in individual incomplete Bernal graphite sections from $N=1$ to 14 . Solid and dashed bars represent the values of middle sections and end sections, respectively. (b) List of the same quantities for multilayer stacks of all possible configurations from a single layer to seven layers. 


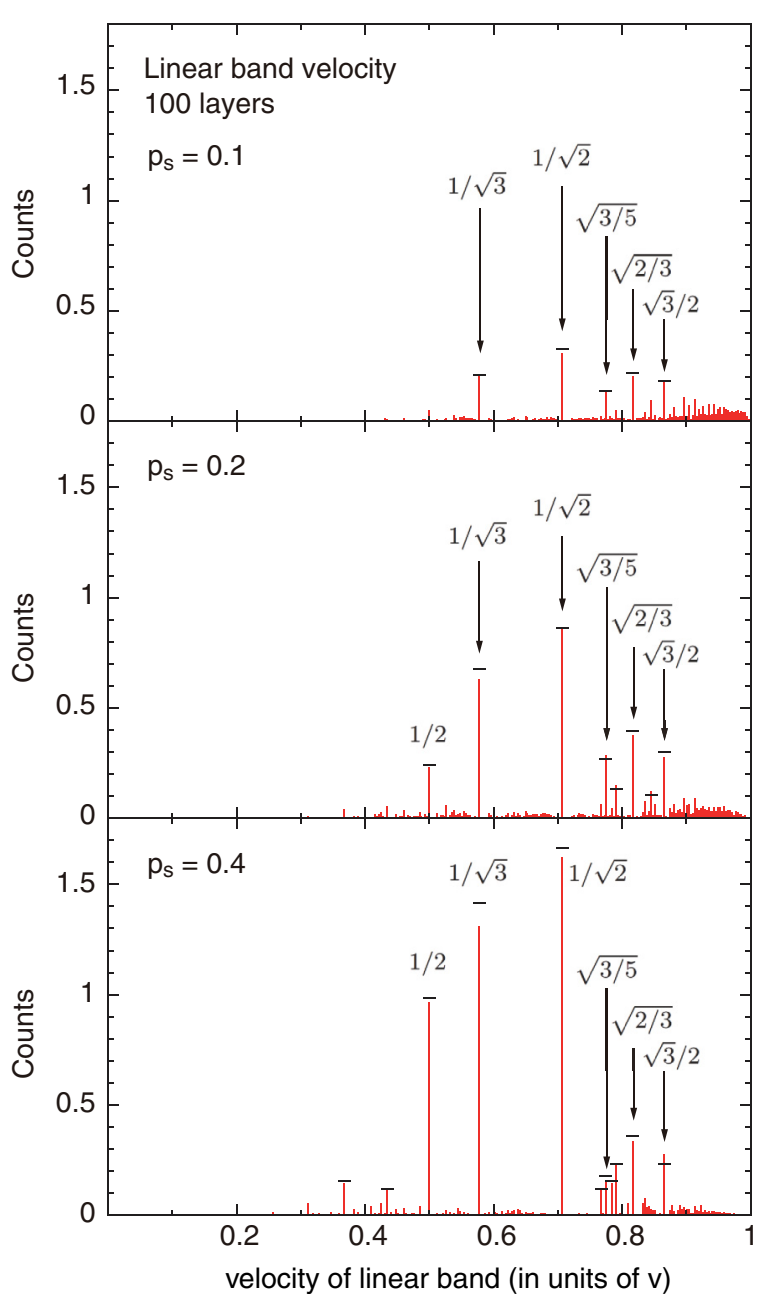

FIG. 8. (Color online) Averaged distribution of the velocity of low-energy linear bands in 100-layer Bernal-rhombohedral mixed graphite with the stacking-fault probability $p_{s}=0.1,0.2$, and 0.4 . Short horizontal bars attached to some major peaks are obtained by the approximation (see the text).

rhombohedral structure is found by a few $10 \%$ out of natural graphite, ${ }^{7}$ we expect $p_{s}$ is typically a few times 0.1 .

Figures 8 and 9 show the distribution of the linear band velocity and quadratic band mass in 100-layer systems averaged over 1000 different configurations, at $p_{s}=0.1,0.2$, and 0.4. The velocities and the masses are again obtained from the eigenenergies of the original Hamiltonian (3) at small momenta. The width of each histogram bin is $0.003 \mathrm{v}$ for velocity, and $0.006\left(\gamma_{1} / 2 v^{2}\right)$ for mass. The height represents the average number of bands per a single 100-layer system. We actually observe the strong peaks at the frequently-appearing values listed in Table I. The relative heights between different peaks depends significantly on $p_{s}$ : in particular, the velocity of $1 / 2$ (in units of $v$ ) is almost absent in $p_{s}=0.1$, but at $p_{s}=0.4$ it is more than half of the tallest peak of $1 / \sqrt{2}$. It might be possible to estimate $p_{s}$ experimentally for a given graphite sample, by comparing the amplitudes of different peaks.

We can approximately estimate the peak heights using simple probability calculation without taking the average of the exact band structure. Let us consider a large multilayer stack composed of $N_{\text {tot }}$ layers, in which the stacking fault

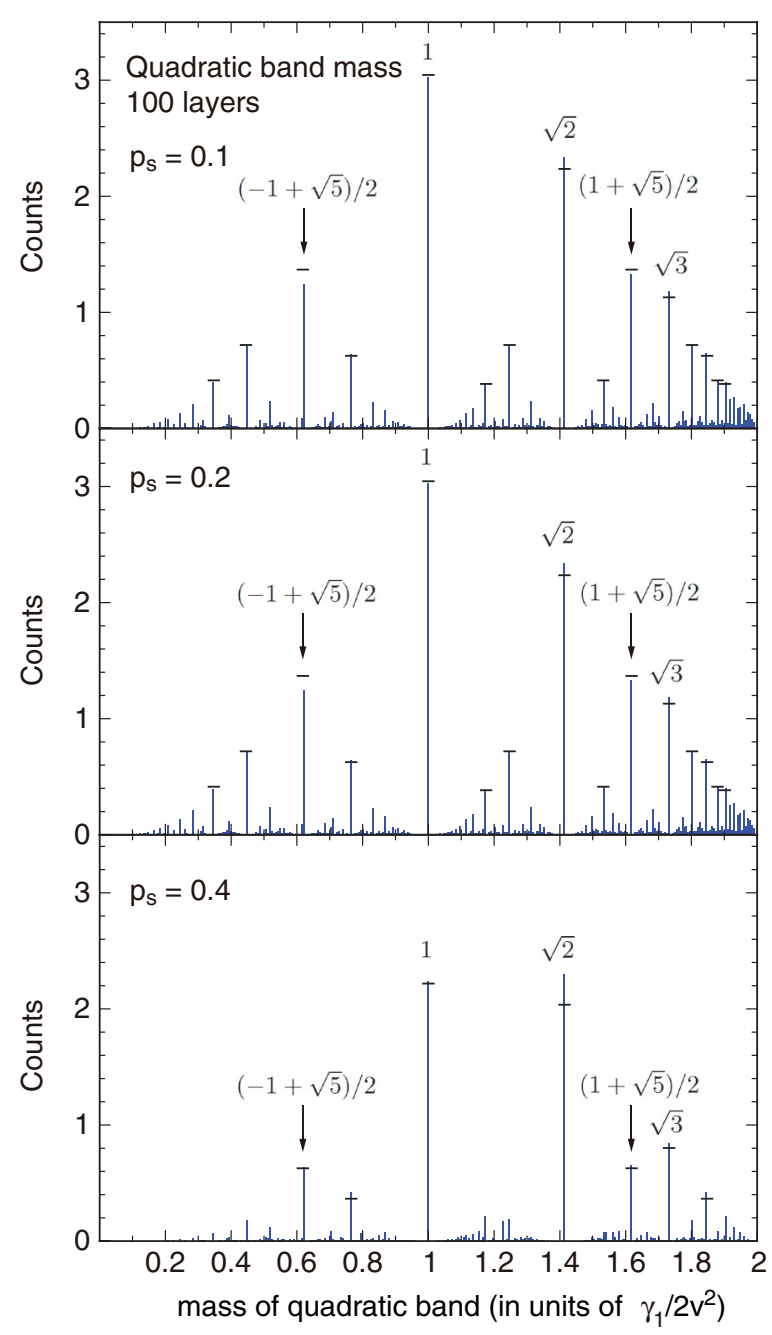

FIG. 9. (Color online) Averaged distribution of effective mass of low-energy quadratic bands similar to Fig. 8.

takes place with probability $p_{s}$. The probability for a given section (surrounded by stacking faults) to have length $N$ is written as

$$
P(N)=p_{s}\left(1-p_{s}\right)^{N-1} .
$$

The averaged length of a section is given by

$$
\bar{N}=\sum_{N=1}^{\infty} N P(N)=\frac{1}{p_{s}},
$$

and the average number of sections included in the whole system is

$$
\bar{M}=\frac{N_{\text {tot }}}{\bar{N}}=p_{s} N_{\text {tot }} .
$$

Now we consider the expected number of a certain sequence $(\circ, a, \circ)$ existing in the whole system. When we look at a particular section, the probability that its length is $a$ and the left- and right-neighboring sections are both odd numbers at the same time, is given by $P_{\text {odd }} P(a) P_{\text {odd }}$, where $P_{\text {odd }}=$ $P(1)+P(3)+P(5)+\cdots=1 /\left(2-p_{s}\right)$. The number of the sequence $(\circ, a, \circ)$ in the system is then obtained by multiplying 
this with the number of sections, $\bar{M}$, namely,

$$
n(\circ, a, \circ) \approx p_{s} N_{\text {tot }} P(a) P_{\text {odd }}^{2} \text {. }
$$

For the end section $(a, \circ)$, similarly, we have

$$
n(a, \circ) \approx 2 P(a) P_{\text {odd }},
$$

where the factor 2 comes from two ends of the whole system. For the combined sequence $(\circ, a, b, \circ), P(a)$ is just replaced with $P(a) P(b)$ in above equations. For the case $(\bullet, a, \bullet)$ appearing in the effective mass table, $P_{\text {odd }}$ is replaced with $1-P(2)$.

The population of a given velocity/mass can be estimated by summing up these numbers over all the corresponding sequences. For example, the number of bands with velocity $1 / \sqrt{3}$ is approximated by $n(\circ, 4, \circ)+n(\circ, 4,2,4, \circ)$, according to Table I(a). We calculate the populations of several representative velocities/masses in this manner, and show the estimated values as short horizontal bars in Figs. 8 and 9. While we took only a finite number of the configurations giving relatively large contributions, the approximation actually works quite well. We note that the peak diagrams like Figs. 8 and 9 are not universal but depend on the total number of layers $N_{\text {tot }}$, because the contribution of the middle section is proportional to $N_{\text {tot }}$ as in Eq. (29), while that of the end section, Eq. (30), is not. In very large multilayer stacks with $N_{\text {tot }}>\sim 1000$, the end section component becomes negligible, and all the peaks just scale in proportion to $N_{\text {tot }}$.

\section{Optical absorption spectrum}

Frequently appearing band structures give rise to striking signals in the optical absorption spectrum. The optical absorption is related to the dynamical conductivity, which is given within the linear response by,

$$
\sigma(\omega)=\frac{e^{2} \hbar}{i S} \sum_{\alpha, \beta} \frac{f\left(\varepsilon_{\alpha}\right)-f\left(\varepsilon_{\beta}\right)}{\varepsilon_{\alpha}-\varepsilon_{\beta}} \frac{\left|\left\langle\alpha\left|v_{x}\right| \beta\right\rangle\right|^{2}}{\varepsilon_{\alpha}-\varepsilon_{\beta}+\hbar \omega+i \delta},
$$

where $S$ is the area of the system, $v_{x}=\partial \mathcal{H} / \partial p_{x}$ is the velocity operator, $\delta$ is the positive infinitesimal, $f(\varepsilon)$ is the Fermi distribution function, and $|\alpha\rangle$ and $\varepsilon_{\alpha}$ describe the eigenstate and the eigenenergy of the system. The transmission of light incident perpendicular to a two-dimensional system is given by $^{42}$

$$
T=\left.\left|1+\frac{2 \pi}{c} \sigma(\omega)\right|\right|^{-2}
$$

Here, we calculate the dynamical conductivity of 100-layer graphites averaged over 1000 different configurations at fixed stacking-fault probability $p_{s}$. We set the Fermi energy at the charge neutral point, $\varepsilon_{F}=0$. Figure 10 shows the dynamical conductivity as a function of frequency $\omega$ calculated for $p_{s}=0,0.2$ and 0.4 . The peaks characterizing the spectrum mainly come from the transition between $\varepsilon=0$ and the band edge of the $\mathrm{C} 1$ band, $\varepsilon=2 \gamma_{1} \cos q_{l}$. The system at $p_{s}=0$ is a pure Bernal-stacked 100-layer graphite, and the absorption edge appears at $\hbar \omega=2 \gamma_{1} \cos q_{l}$ with $q_{l}=l \pi / 101$ $(l=1,2, \ldots, 100)$. Indeed, we see a regular series of small peaks for $\hbar \omega<2 \gamma_{1}$. A major peak at $\hbar \omega=\gamma_{1}$ comes from densely distributed absorption edges at $q_{l} \sim 0$.

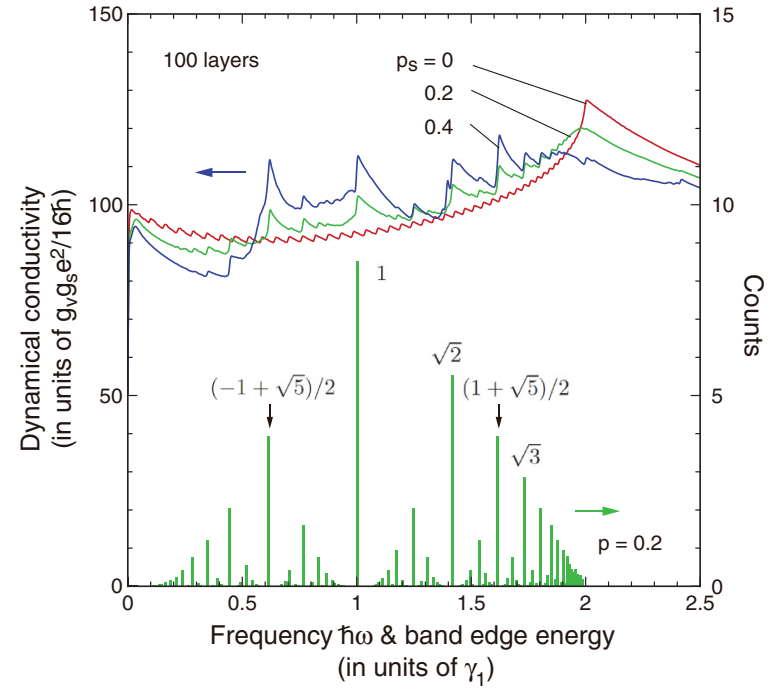

FIG. 10. (Color online) Averaged dynamical conductivity of 100-layer Bernal-rhombohedral mixed graphites with stacking-fault probability $p_{s}=0,0.2$, and 0.4 . Histogram at the bottom is the averaged distribution of the band edges (band energies at zero momentum) in $p_{s}=0.2$.

For finite $p_{s}$, on the other hand, the spectrum is rather regarded as a summation over isolated incomplete graphites of finite layers, as long as the total number of layers is much larger than the averaged length of the Bernal sections. The histogram in Fig. 10 shows the distribution of the energy of C1 band edge $\varepsilon=2 \gamma_{1} \cos q_{l}$ in a 100-layer system at $p_{s}=0.2$. We clearly see that the major peaks appearing in the dynamical conductivity correspond to the absorption edges of frequently appearing band structures.

\section{CONCLUSION}

We studied the electronic structures of general Bernalrhombohedral mixed graphene multilayers with arbitrary configurations. We showed that every low-energy eigenstate is localized in a finite Bernal section bound by rhombohedraltype stacking faults, and the spectrum is well approximated by a collection of the spectra of independent sections, categorized into linear, quadratic, and cubic (or higher-order) bands. We found that the ensemble-averaged spectrum is not smooth, but exhibits a number of discrete structures originating from finite Bernal sections or their combinations, which are statistically likely to appear in a random configuration. In the low-energy region, in particular, there are frequently-appearing linear bands and quadratic bands with specific band velocities or curvatures. In the higher energy region away from the Dirac point, band edges are likely to appear at some particular energies. Those discrete properties may be detected in general graphite samples using experimental techniques such as optical or magnetooptical absorption spectroscopy, and those observations would be useful to probe the stacking structure of graphene multilayers.

While in the present study we took only the relevant hopping parameters $\gamma_{0}$ and $\gamma_{1}$, let us briefly mention expected effects of the neglected parameters. In Bernal graphite, the major additional effects on the zero-energy band structure 
are caused by $\gamma_{2}, \gamma_{3}, \gamma_{4}$, and $\gamma_{5}$. The parameters $\gamma_{2}$ and $\gamma_{5}$ describe the coupling between vertically aligned atoms on the next-nearest neighboring layers. They cause a relative energy shift among the Dirac points of decomposed bilayerlike and monolayer-like subbands, resulting in a semimetallic band structure. $6,26,34,43,44$ Parameter $\gamma_{3}$ describes oblique nearest-layer coupling between $B_{2 n}-A_{2 n \pm 1}$ in Fig. 1(a), and it is responsible for the trigonal warping effect where the energy dispersion in each valley is distorted under a $120^{\circ}$ symmetry. 6,26,27,39 Parameter $\gamma_{4}$ represents similar oblique couplings $A_{n}-A_{n+1}$ and $B_{n}-B_{n+1}$, producing a weak electronhole asymmetry by adding the positive quadratic term to both conduction and valence bands. $6,26,43,44$ The energy range of these additonal effects is typically of the order of a few to $10 \mathrm{meV}$. We expect that the same properties should be shared by incomplete Bernal sections, considering the similarity in the lattice structure. In rhombohedral graphite, on the other hand, the parameter $\gamma_{2}$ works in a different way, contributing to the trigonal warping effect together with $\gamma_{3}$. ${ }^{33}$ This naturally suggests that a similar trigonal warping appears in the F3 band at the rhombohedral stacking fault between Bernal sections.

The electronic structure is also influenced by interlayer potential asymmetry caused by the environment or external electric field, and, to describe these effects correctly, we need to include the screening effect self-consistently. ${ }^{45,46}$ In multilayer graphenes, the charge distribution is generally concentrated near the surface to screen the field, ${ }^{45,46}$ and the screening strength significantly differs between Bernal and rhombohedral stacking. ${ }^{46}$ The field effect of a mixed-stacking structure is expected to lie between the two pure cases, and we leave a detailed analysis as a future problem.

\section{ACKNOWLEDGMENTS}

This project has been funded by JST-EPSRC Japan-UK Cooperative Programme Grant EP/H025804/1 and by Grantsin-Aid for Scientific Research No. 24740193 from JSPS.

\section{APPENDIX A: EIGENENERGIES OF F2 AND F3 STATES}

Here, we show that F2 and F3 states defined in the text are approximate eigenstates with eigenenergies (15) and (16), respectively, in an independent incomplete Bernal graphite. Let us consider the $N$-layer middle section shown in Fig. 3(b), and assign the site indices $a_{j}(j=0,1, \ldots, N)$ and $b_{j}(j=$ $1, \ldots, N-1)$ as in the figure. The Schrödinger equation on the layer $j=1, \ldots, N$ is written as

$$
\begin{gathered}
\varepsilon f\left(b_{j}\right)=v p e^{i \theta_{j}} f\left(a_{j}\right), \\
\varepsilon f\left(a_{j}\right)=v p e^{-i \theta_{j}} f\left(b_{j}\right)+\gamma_{1}\left[f\left(a_{j-1}\right)+f\left(a_{j+1}\right)\right],
\end{gathered}
$$

and on $j=0$ and $N$ as

$$
\varepsilon f\left(a_{0}\right)=\gamma_{1} f\left(a_{1}\right), \quad \varepsilon f\left(a_{N}\right)=\gamma_{1} f\left(a_{N-1}\right),
$$

where $\varepsilon$ is the eigenenergy.

For the F2 state, the exact wave function is given by Eq. (11), plus a correction due to the first order perturbation in the momentum $p$. The wave amplitudes on the free sites are of zeroth order and given as in Eq. (11),

$$
f\left(b_{j}\right)=C e^{i \theta_{j}} \sin q_{l}^{\prime}(j-1),
$$

where $C$ is a constant. The amplitudes on the chained sites are then derived from Eq. (A1) as

$$
f\left(a_{j}\right)=\frac{\varepsilon}{v p} C \sin q_{l}^{\prime}(j-1) .
$$

For $j=1, \ldots, N-1$, we substitute above $f\left(a_{j}\right)$ for Eq. (A2) to find

$$
\varepsilon^{2}=\left(2 \gamma_{1} \cos q_{l}^{\prime}\right) \varepsilon+v^{2} p^{2}
$$

giving

$$
\varepsilon \approx-\frac{v^{2} p^{2}}{2 \gamma_{1} \cos q_{l}^{\prime}}
$$

as long as $v p \ll 2 \gamma_{1} \cos q_{l}^{\prime}$. For the end sites $j=0$ and $j=N$, on the other hand, Eq. (A3) yields to

$$
\frac{\varepsilon^{2}}{v p} \sin q_{l}^{\prime}=0,
$$

which stands within $O\left(p^{2}\right)$ since $\varepsilon \propto p^{2}$.

For F3 states, it is straightforward to show that the exact eigenstates at finite $p$ are explicitly written as

$$
\begin{gathered}
f\left(b_{1}\right)=1, \quad f\left(a_{0}\right)=-\frac{v p e^{-i \theta_{1}}}{\gamma_{1}} ; \quad \text { for }(\mathrm{F} 3, \mathrm{~L}), \\
f\left(b_{N-1}\right)=1, \quad f\left(a_{N}\right)=-\frac{v p e^{-i \theta_{n-1}}}{\gamma_{1}} ; \text { for }(\mathrm{F} 3, \mathrm{R}),
\end{gathered}
$$

and the eigenenergies are exactly zero.

\section{APPENDIX B: INTERSECTION COUPLING BY $N_{i}=2$}

We consider the hybridization of F2 and F3 states in a series of incomplete Bernal sections $\left(N_{i-1}, 2, N_{i+1}\right)$, as illustrated in Fig. 5(b), to derive Eq. (24) for the reconstructed wavenumber $q_{l}^{\prime}$. We assign the site indices $\left(a_{i}, b_{i}\right),\left(a_{i}^{\prime}, b_{i}^{\prime}\right)$, and $\left(a_{i}^{\prime \prime}, b_{i}^{\prime \prime}\right)$ for the section $N_{i-1}, 2$, and $N_{i+1}$, respectively, as shown in Fig. 5(b). We assume either $N_{i \pm 2}$ (out of the figure) is not 2, and then we can neglect the coupling with them.

We consider a small momentum $p \ll \gamma_{1} / v$, and treat it as a perturbation. The zeroth-order wave function for a hybridized state is expressed as a linear combination of F2 and F3 states of the sections $N_{i-1}$ and $N_{i+1}$ having a node at $b_{1}$ and $b_{N_{i+1}-1}^{\prime \prime}$, respectively, and a $\mathrm{C} 1$ state in the middle. Then the wave function is written as

$$
\begin{aligned}
& f\left(b_{j}\right)=C e^{i \theta_{j}} \sin q(j-1), \\
& f\left(b_{j}^{\prime \prime}\right)=C^{\prime \prime} e^{i \theta_{j}^{\prime \prime}} \sin q\left(j-N_{i-1}+1\right), \\
& f\left(a_{0}^{\prime}\right)=-f\left(a_{2}^{\prime}\right)=C^{\prime},
\end{aligned}
$$

where $C, C^{\prime}, C^{\prime \prime}$, and $q$ are quantities to be solved, and $\theta_{j}\left(\theta_{j}^{\prime \prime}\right)$ is defined by $e^{i \theta_{j}}\left(e^{i \theta_{j}^{\prime \prime}}\right)=\left(p_{x} \pm i p_{y}\right) / p$ when $b_{j}\left(b_{j}^{\prime \prime}\right)$ is $B$ and $A$ sites, respectively.

The amplitudes at chained sites $a_{j}$ and $a_{j}^{\prime \prime}$, which are linear in $p$, can be obtained in exactly the same way as in Appendix A as

$$
\begin{aligned}
& f\left(a_{j}\right)=\frac{\varepsilon}{v p} C \sin q(j-1), \\
& f\left(a_{j}^{\prime \prime}\right)=\frac{\varepsilon}{v p} C^{\prime \prime} \sin q\left(j-N_{i-1}+1\right),
\end{aligned}
$$


where $\varepsilon$ is the eigenenergy. The Schrödinger equations at $a_{N_{i-1}}$ and $a_{0}^{\prime \prime}$ are given by

$$
\begin{gathered}
v p e^{-i \theta_{N_{i-1}} f\left(a_{0}^{\prime}\right)+\gamma_{1} f\left(a_{N_{i-1}-1}\right)}=\varepsilon f\left(a_{N_{i-1}}\right), \\
v p e^{-i \theta_{0}^{\prime \prime}} f\left(a_{2}^{\prime}\right)+\gamma_{1} f\left(a_{1}^{\prime \prime}\right)=\varepsilon f\left(a_{0}^{\prime \prime}\right) .
\end{gathered}
$$

By substituting the wave amplitudes, we have

$$
\begin{aligned}
& C^{\prime}=\frac{\varepsilon}{v^{2} p^{2}} C\left[\varepsilon \sin q\left(N_{i-1}-1\right)-\gamma_{1} \sin q\left(N_{i-1}-2\right)\right], \\
& C^{\prime}=-\frac{\varepsilon}{v^{2} p^{2}} C^{\prime \prime}\left[\varepsilon \sin q\left(N_{i+1}-1\right)-\gamma_{1} \sin q\left(N_{i+1}-2\right)\right] .
\end{aligned}
$$

Also, the Schrödinger equations at $a_{0}^{\prime}$ and $a_{2}^{\prime}$,

$$
\begin{aligned}
v p e^{i \theta_{N_{i-1}}} f\left(a_{N_{i-1}}\right)+\gamma_{1} f\left(a_{1}^{\prime}\right) & =\varepsilon f\left(a_{0}^{\prime}\right), \\
v p e^{i \theta_{0}^{\prime \prime}} f\left(a_{0}^{\prime \prime}\right)+\gamma_{1} f\left(a_{1}^{\prime}\right) & =\varepsilon f\left(a_{2}^{\prime}\right),
\end{aligned}
$$

lead to

$$
C^{\prime}=\frac{1}{\sqrt{2}}\left[C \sin q\left(N_{i-1}-1\right)+C^{\prime \prime} \sin q\left(N_{i+1}-1\right)\right] .
$$

The wave number $q$ and the relative wave amplitudes $C^{\prime} / C$ and $C^{\prime \prime} / C$ are obtained by solving Eqs. (B4) and (B6). After some algebra, we obtain the equation for $q=q_{l}^{\prime}$ as Eq. (24). While the argument above is only valid when $N_{i \pm 1} \geqslant 3$, we can show that Eq. (24) stands also when either or both $N_{i \pm 1}$ is 1 or 2.
${ }^{1}$ P. R. Wallace, Phys. Rev. 71, 622 (1947).

${ }^{2}$ J. W. McClure, Phys. Rev. 104, 666 (1956).

${ }^{3}$ J. C. Slonczewski and P. R. Weiss, Phys. Rev. 109, 272 (1958).

${ }^{4}$ J. W. McClure, Phys. Rev. 119, 606 (1960).

${ }^{5}$ G. Dresselhaus and M. S. Dresselhaus, Phys. Rev. 140, A401 (1965).

${ }^{6}$ M. S. Dresselhaus and G. Dresselhaus, Adv. Phys. 51, 1 (2002).

${ }^{7}$ H. Lipson and A. R. Stokes, Proc. Roy. Soc. A 181, 101 (1942).

${ }^{8}$ R. R. Haering, Can. J. Phys. 36, 352 (1958).

${ }^{9}$ J. W. McClure, Carbon 7, 425 (1969).

${ }^{10}$ R. R. Nair, P. Blake, A. N. Grigorenko, K. S. Novoselov, T. J. Booth, T. Stauber, N. M. R. Peres, and A. K. Geim, Science 320, 1308 (2008).

${ }^{11}$ A. B. Kuzmenko, E. van Heumen, F. Carbone, and D. van der Marel, Phys. Rev. Lett. 100, 117401 (2008).

${ }^{12}$ K. F. Mak, M. Y. Sfeir, J. A. Misewich, and T. F. Heinz, PNAS 107, 14999 (2010).

${ }^{13}$ K. F. Mak, J. Shan, and T. F. Heinz, Phys. Rev. Lett. 104, 176404 (2010).

${ }^{14}$ A. C. Ferrari, J. C. Meyer, V. Scardaci, C. Casiraghi, M. Lazzeri, F. Mauri, S. Piscanec, D. Jiang, K. S. Novoselov, S. Roth, and A. K. Geim, Phys. Rev. Lett. 97, 187401 (2006).

${ }^{15}$ C. H. Lui, Z. Li, Z. Chen, P. V. Klimov, L. E. Brus, and T. F. Heinz, Nano Lett. 11, 164 (2011).

${ }^{16}$ J. Ping and M. S. Fuhrer, Nano Lett. 12, 4635 (2012).

${ }^{17}$ M. F. Craciun, S. Russo, M. Yamamoto, J. B. Oostinga, A. F. Morpurgo, and S. Tarucha, Nat. Nanotechnology 4, 383 (2009).

${ }^{18}$ A. Kumar, W. Escoffier, J. M. Poumirol, C. Faugeras, D. P. Arovas, M. M. Fogler, F. Guinea, S. Roche, M. Goiran, and B. Raquet, Phys. Rev. Lett. 107, 126806 (2011).

${ }^{19}$ W. Bao, L. Jing, J. Velasco, Jr, Y. Lee, G. Liu, D. Tran, B. Standley, M. Aykol, S. B. Cronin, D. Smirnov, M. Koshino, E. McCann, M. Bockrath, and C. N. Lau, Nat. Phys. 7, 948 (2011).

${ }^{20}$ L. Zhang, Y. Zhang, J. Camacho, M. Khodas, and I. Zaliznyak, Nat. Phys. 7, 953 (2011)
${ }^{21}$ T. Taychatanapat, K. Watanabe, T. Taniguchi, and P. Jarillo-Herrero, Nat. Phys. 7, 621 (2011).

${ }^{22}$ T. Khodkov, F. Withers, D. C. Hudson, M. F. Craciun, and S. Russo, Appl. Phys. Lett. 100, 013114 (2012).

${ }^{23}$ E. A. Henriksen, D. Nandi, and J. P. Eisenstein, Phys. Rev. X 2, 011004 (2012).

${ }^{24}$ F. Guinea, A. H. Castro Neto, and N. M. R. Peres, Phys. Rev. B 73, 245426 (2006)

${ }^{25}$ S. Latil and L. Henrard, Phys. Rev. Lett. 97, 036803 (2006).

${ }^{26}$ B. Partoens and F. M. Peeters, Phys. Rev. B 74, 075404 (2006).

${ }^{27}$ M. Koshino and T. Ando, Phys. Rev. B 76, 085425 (2007).

${ }^{28}$ M. Aoki and H. Amawashi, Solid State Commun. 142, 123 (2007).

${ }^{29}$ M. Koshino and T. Ando, Phys. Rev. B 77, 115313 (2008).

${ }^{30}$ C. L. Lu, C. P. Chang, Y. C. Huang, J. H. Ho, C. C. Hwang, and M. F. Lin, J. Phys. Soc. Jpn. 76, 024701 (2007).

${ }^{31}$ J. L. Mañes, F. Guinea, and M. A. H. Vozmediano, Phys. Rev. B 75, 155424 (2007).

${ }^{32}$ H. Min and A. H. MacDonald, Phys. Rev. B 77, 155416 (2008).

${ }^{33}$ M. Koshino and E. McCann, Phys. Rev. B 80, 165409 (2009).

${ }^{34}$ D. P. Arovas and F. Guinea, Phys. Rev. B 78, 245416 (2008).

${ }^{35}$ D. P. DiVincenzo and E. J. Mele, Phys. Rev. B 29, 1685 (1984).

${ }^{36}$ G. W. Semenoff, Phys. Rev. Lett. 53, 2449 (1984).

${ }^{37}$ T. Ando, J. Phys. Soc. Jpn. 74, 777 (2005), and references cited therein.

${ }^{38}$ N. H. Shon and T. Ando, J. Phys. Soc. Jpn. 67, 2421 (1998).

${ }^{39}$ E. McCann and V. I. Fal'ko, Phys. Rev. Lett. 96, 086805 (2006).

${ }^{40}$ P. Plochocka, C. Faugeras, M. Orlita, M. L. Sadowski, G. Martinez, M. Potemski, M. O. Goerbig, J.-N. Fuchs, C. Berger, and W. A. de Heer, Phys. Rev. Lett. 100, 087401 (2008).

${ }^{41}$ M. Orlita, C. Faugeras, J. M. Schneider, G. Martinez, D. K. Maude, and M. Potemski, Phys. Rev. Lett. 102, 166401 (2009).

${ }^{42}$ T. Ando, J. Phys. Soc. Jpn. 38, 989 (1975).

${ }^{43}$ M. Koshino and T. Ando, Solid State Commun. 149, 1123 (2009).

${ }^{44}$ M. Koshino and E. McCann, Phys. Rev. B 83, 165443 (2011).

${ }^{45}$ F. Guinea, Phys. Rev. B 75, 235433 (2007).

${ }^{46}$ M. Koshino, Phys. Rev. B 81, 125304 (2010). 